\title{
Does neural input or processing play a greater role in the magnitude of neuroimaging signals?
}

\section{Sam Harris, Myles Jones, Ying Zheng and Jason Berwick*}

Department of Psychology, University of Sheffield, Sheffield, UK

\section{Edited by:}

David Boas, Massachusetts General

Hospital, USA; Massachusetts Institute of Technology, USA; Harvard Medical

School, USA

\section{Reviewed by:}

Hellmuth Obrig, Charité -

Universitätsmedizin Berlin, Germany;

Max Planck Institute of Human

Cognitive and Brain Sciences,

Germany

Maria Angela Franceschini, Harvard

Medical School, USA

\section{${ }^{*}$ Correspondence:}

Jason Berwick, Department of Psychology, University of Sheffield, Western Bank, Sheffield, South YorkShire S10 2TN, UK.

e-mail: j.berwick@sheffield.ac.uk
An important constraint on how hemodynamic neuroimaging signals such as $\mathrm{fMRI}$ can be interpreted in terms of the underlying evoked activity is an understanding of neurovascular coupling mechanisms that actually generate hemodynamic responses. The predominant view at present is that the hemodynamic response is most correlated with synaptic input and subsequent neural processing rather than spiking output. It is still not clear whether input or processing is more important in the generation of hemodynamics responses. In order to investigate this we measured the hemodynamic and neural responses to electrical whisker pad stimuli in rat whisker barrel somatosensory cortex both before and after the local cortical injections of the GABA $_{A}$ agonist muscimol. Muscimol would not be expected to affect the thalamocortical input into the cortex but would inhibit subsequent intra-cortical processing. Pre-muscimol infusion whisker stimuli elicited the expected neural and accompanying hemodynamic responses to that reported previously. Following infusion of muscimol, although the temporal profile of neural responses to each pulse of the stimulus train was similar, the average response was reduced in magnitude by $\sim 79 \%$ compared to that elicited pre-infusion. The whisker-evoked hemodynamic responses were reduced by a commensurate magnitude suggesting that, although the neurovascular coupling relationships were similar for synaptic input as well as for cortical processing, the magnitude of the overall response is dominated by processing rather than from that produced from the thalamocortical input alone.

Keywords: neurovascular coupling, synaptic activity, muscimol, barrel cortex, whisker

\section{INTRODUCTION}

To produce maps of cortical activation modern neuroimaging techniques such as fMRI rely on changes in hemodynamic variables occurring in close proximity to localized increases of neural activity. This relationship, termed neurovascular coupling, is still not completely understood. Following experiments both in anesthetized (Lauritzen, 2001; Jones et al., 2004; Logothetis, 2007; Rauch et al., 2008) and awake animals (Goense et al., 2008), the predominant view is that the hemodynamic response is more closely associated with synaptic activity rather than with spiking output (Lauritzen, 2001; Jones et al., 2004; Logothetis, 2007; Rauch et al., 2008). However, an unresolved issue is whether the hemodynamic response is more representative of the synaptic activity associated with the direct input into an area of cortex or subsequent intra-cortical processing (Mangia et al., 2009). Recent studies in the anesthetized rat have suggested that in both cortical (Radhakrishnan et al., 2007; Franceschini et al., 2008) and sub-cortical (Angenstein et al., 2009) structures, intra-regional processing could be more important than the initial evoked input to a brain region in generating accompanying hemodynamic responses. This has important consequences for the interpretation of data from neuroimaging techniques such as the Blood Oxygen Level dependent (BOLD) fMRI signal as it would allow accurate interpretation of cerebral hemodynamics in terms of stimulus processing. Although these studies (Radhakrishnan et al., 2007; Franceschini et al., 2008; Angenstein et al., 2009) strongly suggested intra-regional processing was more associated with hemodynamic signals than with input, they did not dissociate the two and examine the accompanying hemodynamics. Therefore to test this idea directly it would be advantageous to manipulate a neural system to clearly disassociate the input from the cortical processing. To further this aim, we measured the hemodynamic and neural responses to $16 \mathrm{~s}$ electrical whisker pad stimuli in rat barrel cortex using 2-dimensional optical imaging spectroscopy (2D-OIS) and multi-channel electrophysiology both before and after inactivation of cortical processing by local cortical injection of the $\gamma$-aminobutyric acid type $\mathrm{A}\left(\mathrm{GABA}_{\mathrm{A}}\right)$ receptor agonist muscimol. As based on previous work (Higley and Contreras, 2007) cortical muscimol infusion would not be expected to affect the thalamocortical input into the cortex but inhibit subsequent intracortical processing. This pharmacological manipulation allowed assessment of the separate contributions of cortical input and subsequent processing to the accompanying evoked hemodynamic response. Intra cerebral infusions of this agonist have recently been successfully used to assess the contribution of inhibitory cortical activity to "paired-pulse" whisker inhibition (Higley and Contreras, 2007) and to study the effect of GABAergic tone on neurovascular coupling in the cerebellum (Caesar et al., 2003, 2008). Our data suggest, that although the quantitative neurovascular coupling relationships for cortical input and intra-cortical processing are similar, intra-cortical processing accounts for a the majority of the neurovascular response evoked by sensory stimuli. 


\section{MATERIALS AND METHODS ANIMAL PREPARATION AND SURGERY}

All procedures were performed in accordance with the 1986 Animal (scientific procedures) Act, under approval from the UK Home Office. Female Hooded Lister rats of 230-330 g were kept in a $12-\mathrm{h}$ dark/light cycle at a temperature of $22^{\circ} \mathrm{C}$, with food and water supplied ad libitum. Animals were anesthetized with urethane at $1.25 \mathrm{~g} / \mathrm{kg}$ i.p., additional doses of $0.1 \mathrm{ml}$ urethane were administered if required. Atropine was administered at $0.4 \mathrm{mg} / \mathrm{kg}$ s.c. to lessen mucous secretions during surgery. Temperature was maintained at $37^{\circ} \mathrm{C}$ using a homoeothermic blanket (Harvard Apparatus) through rectal temperature monitoring during surgery and experimental procedures. The animals were tracheotomized, allowing artificial ventilation and recording of end-tidal $\mathrm{CO}_{2}$. Blood gas measurements and end-tidal $\mathrm{CO}_{2}$ measurements were taken to allow correct adjustment of ventilator parameters to keep the animal within physiological limits. Femoral arteries and veins were cannulated to allow the measurement of mean arterial blood pressure (MABP) and drug infusion. Phenylephrine was infused at $0.13-0.26 \mathrm{mg} / \mathrm{h}$ to maintain MABP between 100-110 mmHg (Golanov et al., 1994; Nakai and Maeda, 1999). Animals were placed in a stereotaxic frame (Kopf instruments). The skull overlying the somatosensory cortex was thinned to translucency with a dental drill, with the skull surface cooled with saline. A circular plastic 'well' (20-mm diameter) was attached over the thinned area of the skull using dental cement. The well was filled with saline, to reduce specularities from the skull surface.

\section{2-DIMENSIONAL SPECTROSCOPY (2D-OIS) IMAGING}

A Dalsa $1 \mathrm{M} 30 \mathrm{P}$ camera operating in $4 \times 4$ binning mode recorded images of the cortical surface and each image pixel represented $75 \times 75$ um The camera has a quantum efficiency of $28 \%$ at $500 \mathrm{~nm}$. The cortical surface was illuminated with four wavelengths of light using a Lambda DG-4 high-speed filter changer (Sutter Instrument Company, Novato, CA, USA) and light source that was synchronized to camera image capture. The four wavelengths were specifically chosen as two pairs ( $495 \mathrm{~nm} \pm 31 \mathrm{FWHM}$ and $559 \mathrm{~nm} \pm 16 \mathrm{FWHM} ; 575 \mathrm{~nm} \pm 14 \mathrm{FWHM}$ and $587 \mathrm{~nm} \pm 9$ FWHM). The wavelengths in each pair were chosen such that they had a similar total absorption coefficient and thus sample the same tissue volume but have specific absorption coefficients for oxyhemoglobin $\left(\mathrm{HbO}_{2}\right)$ and deoxyhemoglobin ( $\left.\mathrm{Hbr}\right)$ that are as different as is possible to maximize signal to noise ratio. The frame rate of the camera was $32 \mathrm{~Hz}$, which was synchronized to the filter switching, thereby giving an $8 \mathrm{~Hz}$ effective frame rate for each wavelength and therefore the subsequent estimates of hemodynamics. To create spatio-temporal "maps" of cortical hemodynamics the data were subject to Spectral analysis using a path length scaling algorithm (PLSA) described in detail previously (Berwick et al., 2005, 2008). Briefly the algorithm uses modified Beer-Lambert Law with a path length correction factor. We estimated the concentration of hemoglobin in tissue at a concentration $104 \mu \mathrm{M}$ based on previous measurements (Kennerley et al., 2005) and the saturation was calculated on a pixel by pixel basis (Berwick et al., 2008). The spectral analysis produced 2D images over time, of oxyhemoglobin $\left(\mathrm{HbO}_{2}\right)$, deoxyhemoglobin (Hbr), and total hemoglobin (Hbt).

\section{EXPERIMENTAL PROCEDURES}

Subcutaneous stainless steel stimulation electrodes insulated to within $2 \mathrm{~mm}$ of the tip were inserted in a posterior direction between rows $\mathrm{A} / \mathrm{B}$ and $\mathrm{C} / \mathrm{D}$ of the left whisker pad of the rat ensuring the whole whisker pad was stimulated when electrical stimuli were applied. Stimuli produced no change in $\mathrm{MABP}, \mathrm{pCO}_{2}$ or heart rate suggesting that electrical whisker pad stimuli did not produce any systemic changes that could affect cortical hemodynamics of interest.

\section{Localization of whisker region for electrode placement}

An initial 2D-OIS experiment was performed to localize whisker barrel cortex for accurate placement of a multi-channel electrode. Brief whisker pad stimuli (2s) were presented to localize whisker barrel cortex. Electrical whisker pad stimuli (1.2 mA, 2 s, $5 \mathrm{~Hz})$ were presented for 30 trials with a 26 s inter-stimulus interval. Data from individual trials were averaged and subjected to the spectral analysis. Images of hemodynamic changes were co-registered with images of the cortical surfaces to guide electrode placement. A very small whole in the thinned skull directly above the selected location was made with a dental drill and the bone removed. The dura was then pierced with a 27 gauge needle. A 16-channel electrode coupled with a fluidic probe loaded with Muscimol $(75-\mu \mathrm{m}$ spacing, Neuronexus technologies Inc, concentration of Muscimol $0.125 \mu \mathrm{g}$ per $\mu \mathrm{l}$ ), was inserted normal to the cortical surface, to a depth of $1200 \mu \mathrm{m}$. The probe was coupled to a pre-amp and data acquisition device (Medusa Bioamp, TDT, Florida); using a custom written script in MATLAB (Mathworks).

\section{Experimental paradigm}

Following accurate electrode placement, concurrent electrophysiological measures of neural activity were recorded while accompanying cortical hemodynamics were measured simultaneously with 2D-OIS. Once the electrode was inserted a control experiment was performed which consisted of 30 trials with an inter-stimulus interval of $70 \mathrm{~s}$. Stimulation occurred after $19 \mathrm{~s}$ in each trial $(1.2 \mathrm{~mA} 16 \mathrm{~s}$ at $5 \mathrm{~Hz}$ ). Data was trial averaged and then subjected to the same spectroscopy analysis as described above. Simultaneously neural activity was collected across all 16 channels of the multi-electrode at a sampling frequency of $6.1 \mathrm{KHz}$ for a period of $26.7 \mathrm{~s}$ starting $5.2 \mathrm{~s}$ before stimulation onset within each trial. After this experiment $1 \mu \mathrm{l}$ of muscimol was infused over a period of 1 min using a $10-\mu l$ Hamilton syringe and syringe pump (World Precision Instruments Inc). After the muscimol had been allowed to take effect the long stimulation 2D-OIS and simultaneous neural activity paradigm was repeated.

\section{Electrophysiological data analysis}

The 16-channel neural data was analyzed by performing current source density (CSD) analysis. Recordings were averaged over trials, with stimulus onset "jittered" within a 20 -ms window to reduce effects of $50 \mathrm{~Hz}$ mains noise. The resultant evoked field potential recordings were sampled at $6 \mathrm{kHz}$ with 16-bit resolution. The CSD analysis has been described in detail previously (Martindale et al., 2003). The field potentials were used to obtain spatio-temporal estimates of the current sources and sinks within the cortical layers (Nicholson and Freeman, 1975; Mitzdorf, 1985). The current sink 
with the greatest amplitude was located $\sim 500 \mu \mathrm{m}$ below the surface of the brain. This corresponds to layer IV, which receives direct somatosensory input from the ventral posterior thalamus. The main current sink in layer IV, which is believed to be a result of excitatory post synaptic activity (EPSP) in the barrel, was taken as our neural activity measure and will be referred to as the CSD Sink.

\section{Post-mortem cytochrome oxidase histology to confirm location of somatosensory cortex}

Following most experiments post-mortem brain tissue was prepared for subsequent histological analysis. The histological methods have been explained previously (Zheng et al., 2001) and as such is only described briefly here. Rats were transcardially perfused with saline, followed by $4 \%$ paraformaldehyde and finally photographic emulsion (Jessops Ltd) to enable visualization of surface cortical vessels in histogical sections. Brains were removed and the right cortex was separated from the rest of the cerebrum and compressed to a thickness of $2 \mathrm{~mm}$. A cryostat was used to section the cortex into slices tangential to its surface. An initial surface slice of $200 \mu \mathrm{m}$ was sectioned to ensure visualization of the surface blood vessels, and then subsequent $50-\mu \mathrm{m}$ sections were taken. The slices were placed into an incubation medium in a dark room at $37^{\circ} \mathrm{C}$ to allowing stain for cytochrome oxidase to visualize barrel representations; this is a modified version of the procedure described by Wong-Riley and Welt (1980). The photographic emulsion in the vessels in histological sections was developed after staining. Photomicrographs of the resultant histological sections of cortex were taken and the images were linearly warped to each other by locating corresponding features by eye.

\section{RESULTS \\ SPATIAL ANALYSIS OF THE HEMODYNAMIC RESPONSE BEFORE AND AFTER MUSCIMOL INJECTION}

Hemodynamic responses were recorded from the right somatosensory whisker barrel cortex of urethane anesthetized rats following presentation of $16 \mathrm{~s}$ electrical stimulation of the left whisker pad. The electrode (with fluidic port attached) placement was accurately placed into the cortex as demonstrated by an in vivo CCD camera "gray-level" image of the cortical surface (Figure 1A). An image of combined photomicrographs of postmortem histology of the cortical surface and the underling cortical barrels is shown for comparison (Figure 1B). It can be seen the electrode and fluidic port have been inserted into the cortex in the region of whisker barrel $\mathrm{B} 1$. For this representative animal two regions of interest (ROI) were chosen from which to examine the resultant time series of cortical hemodynamics following pixel averaging. One ROI was selected near to the electrode tip and a second from anterior whisker barrels distal to electrode placement. To assess the spatial extent of the hemodynamic response prior and post muscimol infusion, an average response image was created by averaging all images collected during the entire stimulus presentation period (16 s) for $\mathrm{Hbt}, \mathrm{HbO}_{2}$ and $\mathrm{Hbr}$ (Figures 1C,E). For the whisker-evoked hemodynamics collected prior to muscimol infusion (control, Figure 1C) a large increase over the whole whisker barrel cortical region can be observed for both $\mathrm{Hbt}$ and $\mathrm{HbO}_{2}$; whereas $\mathrm{Hbr}$ showed a large decrease over the whole whisker region particularly in the "draining" cortical veins. Examination of the time series of the response for the two ROI selected (Figure 1D) demonstrates that the response in the anterior barrels (dotted lines) distal to the electrode is only slightly larger than from the region immediately surrounding the electrode tip (solid lines). Following infusion of muscimol the spatial extent and magnitude of the hemodynamic response elicited by whisker stimuli was altered compared to that observed prior to muscimol infusion. By observation of spatial images calculated by averaging images collected during stimulus presentation (Figure 1E), $\mathrm{Hbt}, \mathrm{HbO}_{2}$ and $\mathrm{Hbr}$ around the electrode tip were attenuated compared to those elicited prior to muscimol infusion and with respect to the ROI distal to the electrode. This can also be observed in the time series responses from the two ROI (Figure 1F) which demonstrate that the hemodynamic response around the electrode tip has been substantially reduced following muscimol infusion whereas the response from the distal region of interest being relatively unaffected.

\section{TIME SERIES OF ANIMAL AVERAGED CORTICAL HEMODYNAMIC RESPONSES ( $\mathbf{N}=\mathbf{8}$ )}

A region of interest was selected around the electrode tip in each animal Hemodynamic data was pixel averaged to create a time series for each animal and then data was subsequently averaged across animals (Figure 2). The "control" hemodynamic response elicited by $16 \mathrm{~s}$ whisker stimuli prior to muscimol infusion was similar to that described previously (Berwick et al., 2005). It consisted of an immediate large increase in $\mathrm{Hbt}$ and $\mathrm{HbO}_{2}$ that peaked after $\sim 5 \mathrm{~s}$, follow by a decrease to an elevated plateau that lasted for the remainder of stimulus presentation before returning to baseline. The Hbr response was characterized by a small increase within the first second of stimulation, usually referred to as the "deoxy dip" followed by a large decrease below baseline reaching a minima at $\sim 5$ s slowly returning to baseline after stimulus cessation. Despite displaying a similar time course to that elicited by whisker stimuli prior to muscimol infusion, following muscimol infusion all aspects of the hemodynamic were substantially attenuated in magnitude (Figure 2).

\section{Neural activity assessed by current source density analysis (CSD)}

By using a combined multi-channel electrode and infusion fluidic port we were able to monitor the neural response locally at the site of muscimol infusion. CSD analysis converts laminar field potentials evoked by whisker stimuli into current sinks and sources as a function of cortical depth (Nicholson and Freeman, 1975; Mitzdorf, 1985). The neural response to whisker stimuli for a representative animal in the pre-muscimol infusion "control" condition (Figure 3A) showed the characteristic evoked superficial source with an accompanying underlying sink centered at Layer IV. The 'heat map' representation of the CSD analysis was derived by averaging of all 80 impulses ( 16 s stimulation at $5 \mathrm{~Hz}$ ) of the electrical whisker pad stimulus train presented. This current sink in layer IV represents active excitatory post synaptic potentials in response to each electrical whisker pad impulse. We selected this current sink as our measure of neural activity and averaged across animals. (Figure 3B). It can be seen the largest neural response was elicited by the first impulse of the stimulus train with responses to subsequent impulses of the train being at 
A

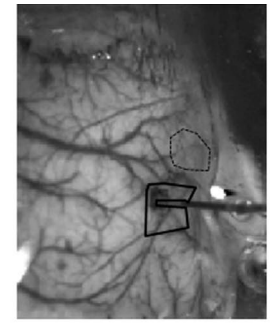

B

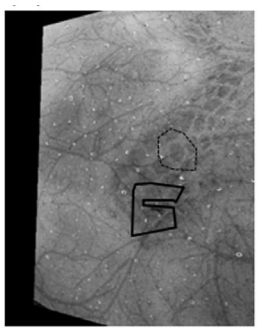

\section{Control - Pre-muscimol injection}

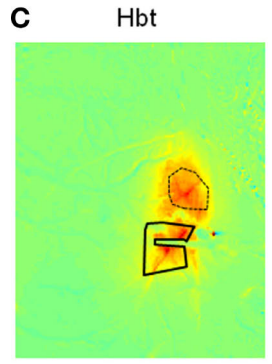

$\mathrm{Hbr}$

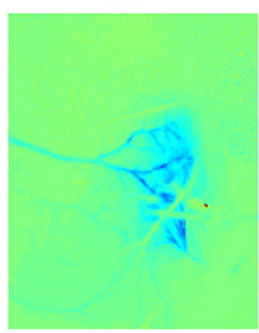

$\mathrm{HbO}_{2}$

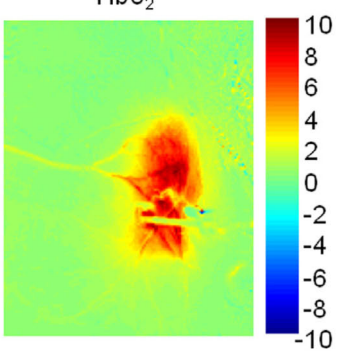

D

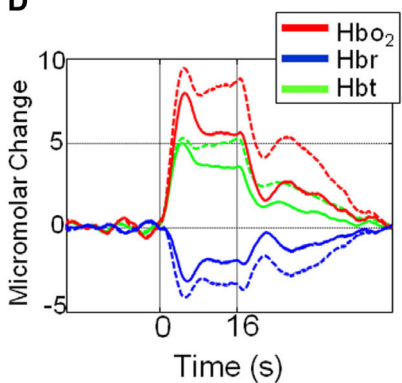

\section{Post muscimol Injection}

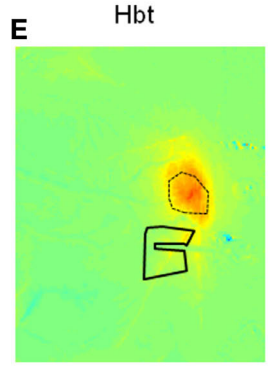

$\mathrm{Hbr}$
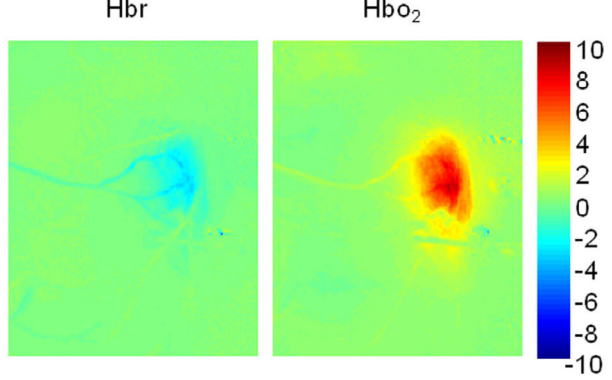

$\mathbf{F}$

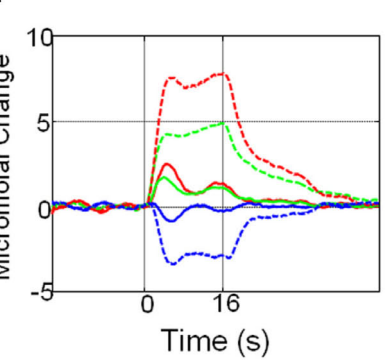

FIGURE 1 | Hemodynamic responses in a representative animal before and after local cortical infusion of muscimol. (A) In vivo gray level CCD camera image of the somatosensory cortical surface. The electrode with drug infusion probe attached is visible on the right hand side of the image. (B) An image of combined photomicrographs of post-mortem histological sections of the cortical surface and the underling cortical barrels from Layer IV. (C) Total blood volume $(\mathrm{Hbt})$, Deoxyhemoglobin $(\mathrm{Hbr})$ and Oxyhemoglobin $\left(\mathrm{HbO}_{2}\right)$ responses to 16-s electrical stimulation of the whisker pad. Each image represents an average change in micromolar concentration from baseline over the 16-s stimulation period. (D) Time series of hemodynamics response prior to muscimol infusion from a region of interest around the electrode tip (solid lines) and an additional $\mathrm{ROI}$ distal to the electrode in the more anterior whisker barrels (dotted lines). (E) Hemodynamic response images post muscimol injection. (F) Time series of hemodynamics response post muscimol infusion from a region of interest around the electrode tip (solid lines) and an additional ROI distal to the electrode in the more anterior whisker barrels (dotted lines). a lower but similar magnitude. Following infusion of muscimol the spatial profile of the results of CSD analysis are similar to that observed prior to muscimol infusion but are greatly reduced in magnitude (Figure 3C). The sink in layer IV appeared to be less broad in time than the control response. Examination of the animal averaged time series of the layer IV sink (Figure 3D) showed that the response to the first impulse of the stimulus train was reduced compared to that elicited prior to muscimol infusion. There was also a large reduction in magnitude of responses to subsequent impulses of the stimulus train compared to the control condition. When these "CSD-sink time series" were averaged across each impulse of the stimulus train (Figure 3E) it can be seen that the size of the sinks elicited were smaller and displayed more of a temporally transient nature compared those elicited prior to muscimol infusion.

\section{Statistical analysis}

The sum $(\Sigma)$ of the time series of Hbt and the layer IV CSD-sink were calculated for each animal (Table 1). As total hemoglobin concentration (Hbt) is the sum of oxyhemoglobin and deoxyhemoglobin concentration and during the hemodynamic response function the increases in Hbo and decreases in Hbr are correlated (Jones et al., 2001, 2002) it was decided to select the magnitude of Hbt changes as the dependent variable for statistical analysis of hemodynamics. A one-way repeated measures (pre or post muscimol injection) MANOVAs with two dependent variables (Hbt and CSD magnitude) suggested that the injection of muscimol caused a significant change in response size (size: Wilks' Lambda $=0.15$, hypothesis $\mathrm{df}=2$, error $\mathrm{df}=6$, $F=16.93, P=0.003)$. Subsequent univariate's ANOVA suggested that both $\mathrm{Hbt}$ and CSD responses were significantly reduced 

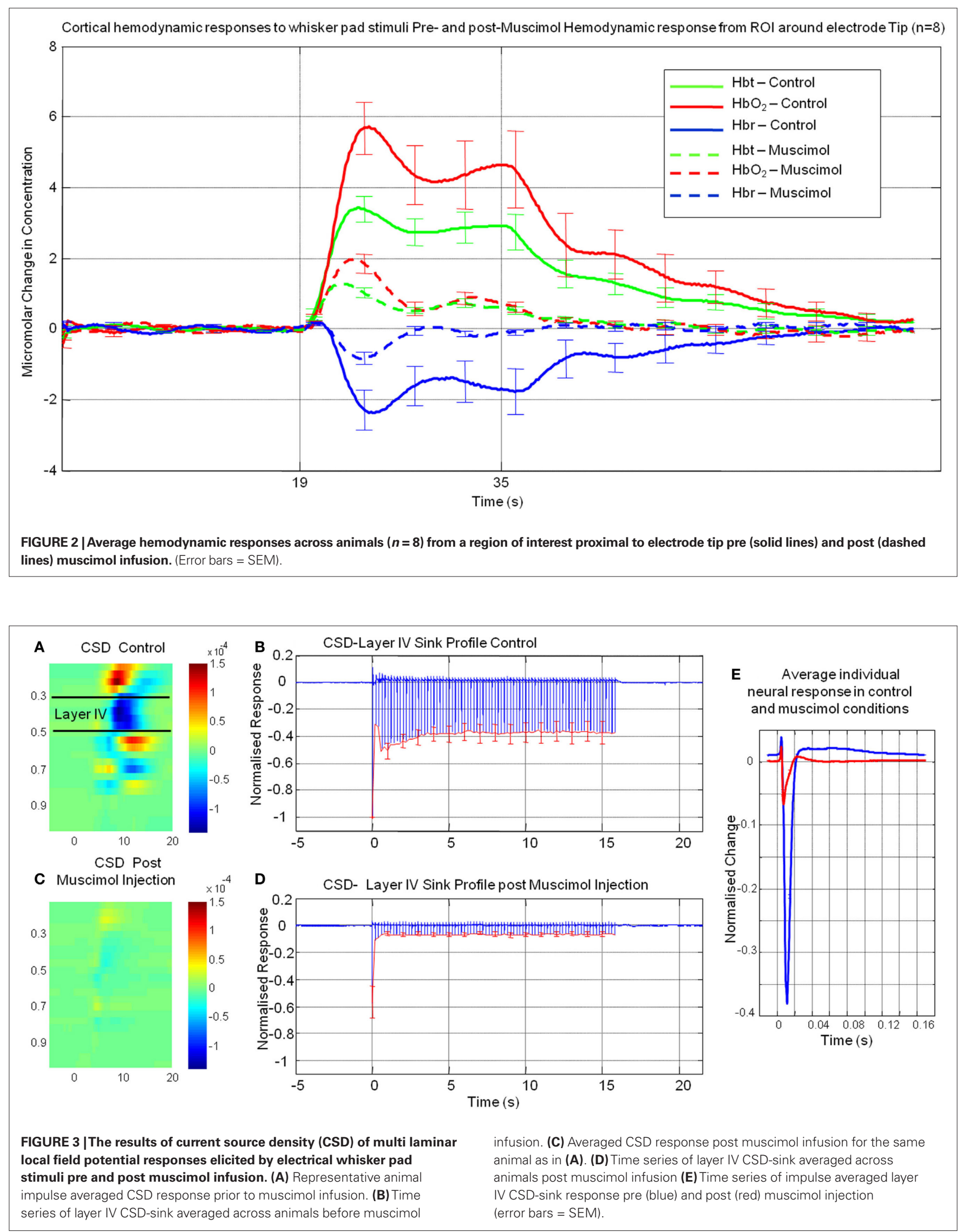


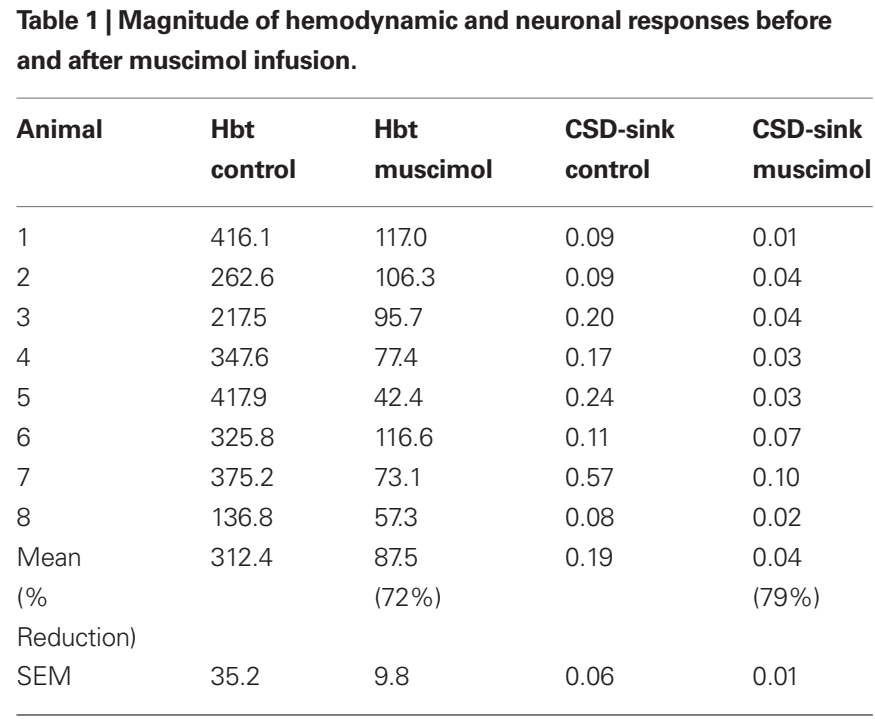

even if Bonferroni corrected. (Hbt: $F=39.487, P=<0.0001$; CSD: $F=8.694, P=0.021)$. The reductions in neural and hemodynamics responses were similar (79 and $72 \%$ respectively). Therefore even though the magnitude of response has reduced, the quantitative relationships of neurovascular coupling were similar before and after muscimol infusion suggesting the neurovascular coupling mechanisms are conserved for input and processing.

\section{DISCUSSION}

The aim of the current study was to assess the neurovascular relationships for input and cortical processing and whether they had equal contributions to the overall neurovascular response elicited by sensory stimuli. We first performed a $16 \mathrm{~s}$ whisker stimulation in control conditions and then repeated the same stimulation following local cortical infusion of the $\mathrm{GABA}_{\mathrm{A}}$ receptor agonist muscimol. Muscimol was infused to prevent the whisker stimulus driven thalamocortical information being processed by further by the cortex (Higley and Contreras, 2007).

The time series of current sink in layer IV following muscimol infusion (Figure 3 far right panel) was far more transient than that elicited by whisker stimuli prior to infusion. It has been shown that, in addition to the synaptic activity due to thalamic input, whisker stimuli also evoke longer latency synaptic activity in layer IV due to cortical interactions. Thus, it appears that it is this longer latency synaptic activity associated with intra-cortical processing that is (Ebner and Armstrong-James, 1990) absent from the time series of CSD-sink following muscimol infusion. Furthermore, Higley and Contreras (2007) showed a similar change in time series of CSD from layer IV evoked by whisker stimuli following intra-cortical musicmol infusion. They made recordings from thalamus and suggested that the thalamocortical input was unchanged by intra-cortical muscimol infusion. This is also suggestive the sink at layer IV provides a measure of intra-cortical processing and thalamic input, the former being disrupted by muscimol infusion.
The data suggests that although the neurovascular relationships were similar between input and processing a vast majority of the magnitude of the response was due to processing. Most studies investigating neurovascular coupling with fMRI or other techniques have concluded that the hemodynamic response is due to synaptic input and cortical processing but have not tried to disassociate the two. Two recent findings have, like this study suggested that the hemodynamic response is due to the processing of information rather than the input. Franceschini et al. (2008) measured the somatosensory evoked potentials (SEPs) evoked by forepaw stimuli and accompanying cortical hemodynamics with diffuse optical imaging tomography. They found that the N1 and $\mathrm{P} 2$ aspects of the field potential predicted the evoked hemodynamics better than did the P1. As P1 is more associated with synaptic input than the N1 and P2 measures of the field potential they suggested that the hemodynamic response was more associated with cortical processing than synaptic input. Angenstein et al. (2009) directly electrically stimulated the perforant pathway of the hippocampus in anesthetized rats and measured spiking output and the accompanying BOLD fMRI signal. They found that perforant pathway stimuli that evoked the same spiking output in hippocampus had variable accompanying BOLD responses and thus the variability in the BOLD response must have been due to differences in intra-regional processing. They therefore concluded that the evoked BOLD response depended more on the local processing of neural activity than the output or input activity. The current study confirms and extends these recent findings by measuring more local changes of neurovascular coupling (Layer IV current sink and local changes in hemoglobin), and by actively disassociating between the synaptic activity associated with input and intra-cortical processing with the use of muscimol infusion.

The results of the present investigation differ from those obtained in studies performed in rat cerebellum (Caesar et al., 2003, 2008) following local muscimol infusion. In these studies muscimol application had little effect on field potential magnitude whereas the blood flow responses were reduced at superficial cerebellar cortical depths. These authors suggested that GABAergic tone, at least in the cerebellum, plays a role in the relay of excitatory input into blood flow responses. Comparison of these results and those of the current study highlight the potential differences in neurovascular responses between different brain areas.

\section{LIMITATIONS OF CURRENT STUDY}

The main limitation of the current study is the assumption that the input from the thalamus is not affected by cortical application of muscimol. This is an important issue because there are far greater corticothalamic descending projections than the thalamocortical projections ascending into the cortex. Recently Higley and Contreras (2007) investigated whether effective cortical neural inhibition following presentation of stimuli to two whiskers was generated in the cortex or a property of sub-cortical interactions. Their experiment was similar that conducted here in that, CSD analysis of neural responses to whisker stimulus were observed before and after cortical application of muscimol (CSD responses also very similar cf Figure 2A, Higley and 
Contreras, 2007). However, unlike the present investigation they also made concurrent electrophysiological measurements of thalamic neural activity and showed that the cortical application of muscimol had no effect on the whisker-evoked responses in the thalamus. This provides good evidence that in our study that the thalamic input to the cortex would be unaffected by muscimol application to the cortex.

\section{REFERENCES}

Angenstein, F., Kammerer, E., and Scheich, H. (2009). The BOLD response in the rat hippocampus depends rather on local processing of signals than on the input or output activity. A combined functional MRI and electrophysiological study. J. Neurosci. 29, 2428-2439.

Berwick, J., Johnston, D., Jones, M., Martindale, J., Martin, C., Kennerley, A. J., Redgrave, P., and Mayhew, J.E. (2008). Fine detail of neurovascular coupling revealed by spatiotemporal analysis of the hemodynamic response to single whisker stimulation in rat barrel cortex. J. Neurophysiol. 99, 787-798.

Berwick, J., Johnston, D., Jones, M., Martindale, J., Redgrave, P., McLoughlin, N., Schiessl, I., and Mayhew, J. E. (2005). Neurovascular coupling investigated with twodimensional optical imaging spectroscopy in rat whisker barrel cortex. Eur. J. Neurosci. 22, 1655-1666.

Caesar, K., Offenhauser, N., and Lauritzen, M. (2008). Gamma-aminobutyric acid modulates local brain oxygen consumption and blood flow in rat cerebellar cortex. J. Cereb. Blood Flow Metab. 28, 906-915.

Caesar, K., Thomsen, K., and Lauritzen, M. (2003). Dissociation of spikes, synaptic activity, and activity-dependent increments in rat cerebellar blood flow by tonic synaptic inhibition. Proc. Natl. Acad. Sci. U.S.A. 100, 16000-16005.

Ebner, F. F., and Armstrong-James, M. A. (1990). Intracortical processes regulating the integration of sensory information. Prog. Brain Res. 86, 129-141.

Franceschini, M. A., Nissila, I., Wu, W., Diamond, S. G., Bonmassar, G., and Boas, D. A. (2008). Coupling between somatosensory evoked potentials and hemodynamic response in the rat. Neuroimage 41, 189-203.

Goense, J. B., Ku, S. P., Merkle, H., Tolias, A. S., and Logothetis, N. K. (2008). fMRI of the temporal lobe of the awake monkey at 7 T. Neuroimage 39, 1081-1093. Golanov, E. V., Yamamoto, S., and Reis, D. J. (1994). Spontaneous waves of cerebral blood flow associated with a pattern of electrocortical activity. Am. J. Physiol. 266, R204-R214.

Higley, M. J., and Contreras, D. (2007). Cellular mechanisms of suppressive interactions between somatosensory responses in vivo. J. Neurophysiol. 97, 647-658.

Jones, M., Berwick, J., Johnston, D., and Mayhew, J. (2001). Concurrent optical imaging spectroscopy and laserDoppler flowmetry: the relationship between blood flow, oxygenation, and volume in rodent barrel cortex. Neuroimage 13, 1002-1015.

Jones, M., Berwick, J., and Mayhew, J. (2002). Changes in blood flow, oxygenation, and volume following extended stimulation of rodent barrel cortex. Neuroimage 15, 474-487.

Jones, M., Hewson-Stoate, N., Martindale, J., Redgrave, P., and Mayhew, J. (2004). Nonlinear coupling of neural activity and CBF in rodent barrel cortex. Neuroimage 22, 956-965.

Kennerley, A. J., Berwick, J., Martindale, J., Johnston, D., Papadakis, N. and Mayhew, J. E., (2005). Concurrent fMRI and optical measures for the investigation of the hemodynamic response function. Magn. Reson. Med. 54, 354-365.

Lauritzen, M. (2001). Relationship of spikes, synaptic activity, and local

\section{CONCLUSION}

The results of this study show that although the quantitative neurovascular coupling relationship is similar for both cortical input and intra-cortical processing a great majority of the measured neurovascular response is driven by intra-cortical processing. These results may have important implications for the interpretation and understanding of cortical fMRI signals.

changes of cerebral blood flow. J. Cereb. Blood Flow Metab. 21, 1367-1383.

Logothetis, N. K. (2007). The ins and outs of fMRI signals. Nat. Neurosci. 10, 1230-1232.

Mangia, S., Giove, F., Tkac, I., Logothetis, N. K., Henry, P. G., Olman, C. A., Maraviglia, B., Di Salle, F., and Ugurbil, K. (2009). Metabolic and hemodynamic events after changes in neuronal activity: current hypotheses, theoretical predictions and in vivo NMR experimental findings. J. Cereb. Blood Flow Metab. 29, 441-463.

Martindale, J., Mayhew, J., Berwick, J., Jones, M., Martin, C., Johnston, D., Redgrave, P., and Zheng, Y. (2003). The hemodynamic impulse response to a single neural event. J. Cereb. Blood Flow Metab. 23, 546-555.

Mitzdorf, U. (1985). Current sourcedensity method and application in cat cerebral cortex: investigation of evoked potentials and EEG phenomena. Physiol. Rev. 65, 37-100.

Nakai, M., and Maeda, M. (1999). Scopolamine-sensitive and resistant components of increase in cerebral cortical blood flow elicited by periaqueductal gray matter of rats. Neurosci. Lett. 270, 173-176.

Nicholson, C., and Freeman, J. A. (1975). Theory of current source-density analysis and determination of conductivity tensor for anuran cerebellum. $J$. Neurophysiol. 38, 356-368.

Radhakrishnan, I., Nissila, I., Wu, W. Diamond, S. G., Bonmassar, G., Boas, D. A., and Franceschini, M. A. (2007). Studying the neurovascular coupling with evoked potentials and hemodynamic signals. Soc. Neurosci. 316.4 .
Rauch, A., Rainer, G., and Logothetis, N. K. (2008).Theeffect of aserotonin-induced dissociation between spiking and perisynaptic activity on BOLD functional MRI. Proc. Natl. Acad. Sci. U.S.A. 105, 6759-6764.

Wong-Riley, M. T., and Welt, C. (1980). Histochemical changes in cytochrome oxidase of cortical barrels after vibrissal removal in neonatal and adult mice. Proc. Natl. Acad. Sci. U.S.A. 77, 2333-2337.

Zheng, Y., Johnston, D., Berwick, J., and Mayhew, J. (2001). Signal source separation in the analysis of neural activity in brain. Neuroimage 13, 447-458.

Conflict of Interest Statement: The authors declare that the research was conducted in the absence of any commercial or financial relationships that could be construed as a potential conflict of interest.

Received: 22 January 2010; paper pending published: 30 March 2010; accepted: 25 June 2010; published online: 11 August 2010.

Citation: Harris S, Jones $M$, Zheng $Y$ and Berwick J (2010) Does neural input or processing play a greater role in the magnitude of neuroimaging signals? Front. Neuroenerg. 2:15. doi: 10.3389/ fnene.2010.00015

Copyright (c) 2010 Harris, Jones, Zheng and Berwick. This is an open-access article subject to an exclusive license agreement between the authors and the Frontiers Research Foundation, which permits unrestricted use, distribution, and reproduction in any medium, provided the original authors and source are credited. 